\title{
Novos aportes teórico-metodológicos para o diagnóstico de osteoartrose em séries esqueléticas e sua importância para a arqueologia brasileira: I - Registro dos processos tafonômicos e dos marcadores ósseos \\ New theoretical and methodological contributions to osteoarthrosis diagnosis in skeletal series and its importance to the Brazilian archaeology: I - Taphonomic processes and bone markers record
}

\author{
Andrea Lessa \\ Universidade Federal do Rio de Janeiro/Museu Nacional. Rio de Janeiro, Rio de Janeiro, Brasil
}

\begin{abstract}
Resumo: Estudos de Marcadores de Estresse Ocupacional (MEO) têm sido cada vez mais realizados com vistas ao entendimento do estilo de vida de populações antigas, uma vez que são potencialmente capazes de informar acerca das solicitações mecânicas ocorridas ao longo da vida, principalmente aquelas relacionadas a atividades cotidianas. Osteoartroses estão entre os principais tipos de $\mathrm{MEO}$, as quais, devido à alta frequência entre populações pré-coloniais, têm sido objeto de muitos estudos bioarqueológicos entre pesquisadores norte-americanos e europeus. Apesar de demonstrado seu potencial para informar sobre padrões adaptativos e divisão sexual e/ou social de tarefas, esses estudos ainda são raros no Brasil. Esta situação tem relação, provavelmente, com uma série de problemas metodológicos para entendimento, identificação e registro dos marcadores osteológicos da osteoartrose. Um amplo projeto desenvolvido pela autora vem evidenciando a falta de padronização nesses estudos, além da utilização de metodologias inadequadas, as quais resultam em erros sistemáticos de quantificação. Como consequência, observa-se a inviabilização de comparações de resultados entre séries afins, além do comprometimento da confiabilidade e interpretação dos padrões observados. Este artigo discute a importância dos critérios de eleição das articulações a serem examinadas, da padronização e adequação do registro dos marcadores ósseos, assim como dos estudos de MEO, especificamente de osteoartroses, para a arqueologia brasileira.
\end{abstract}

Palavras-chave: Bioarqueologia. Metodologia. Diagnóstico. Osteoartrose. Séries esqueléticas.

\begin{abstract}
Studies on Occupational Stress Markers (OSM) have increasingly been performed in order to understand the lifestyle of ancient populations, since they are potentially capable of providing information on mechanical stresses that occur throughout life, especially those related to daily activities. Osteoarthrosis are among the main types of OSM, which, due to the high frequency in pre-colonial populations, have been subject of many bioarchaeological studies among Americans and Europeans researchers. Despite its potential to inform about adaptive patterns and sexual and/or social division of labour have been demonstrated, studies on osteoarthrosis are still rare in Brazil. This situation is probably related to methodological problems for understanding, identifying and registering osteological markers of osteoarthrosis. A broad project developed by the author is highlighting the lack of standardization in these studies, as well as the use of inappropriate methodologies, which result in systematic errors of quantification. As a consequence, the comparison of results among related series is not feasible, besides the questionable reliability and interpretation of observed patterns. This article discusses the importance of the selection criteria of joints to be examined and the standardization and adequacy of the record of bone markers, as well as the studies on OSM, specifically osteoarthrosis, to Brazilian archaeology.
\end{abstract}

Keywords: Bioarchaeology. Methodology. Diagnostic. Osteoarthrosis. Skeletal series.

LESSA, Andrea. Novos aportes teórico-metodológicos para o diagnóstico de osteoartrose em séries esqueléticas e sua importância para a arqueologia brasileira: I - Registro dos processos tafonômicos e dos marcadores ósseos. Boletim do Museu Paraense Emílio Goeldi. Ciências Humanas, v. 8, n. 3, p. 567-583, set.-dez. 2013.

Autor para correspondência: Andrea Lessa. Universidade Federal do Rio de Janeiro/Museu Nacional. Quinta da Boa Vista, s/n - São Cristóvão. Rio de Janeiro, RJ, Brasil. CEP 20940-040 (lessa.mn@gmail.com).

Recebido em 13/12/2012

Aprovado em 13/10/2013

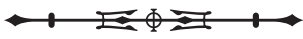




\section{INTRODUÇÃO}

As atividades cotidianas constituem-se como foco cada vez mais explorado nos estudos de paleoepidemiologia, principalmente em populações pré-coloniais. Sua associação intrínseca com os contextos materiais e ambientais proporciona uma abordagem integrativa dos dados arqueológicos e bioarqueológicos, potencialmente informativa sobre estilos de vida e padrões adaptativos.

Sob a perspectiva paleoepidemiológica, as inferências sobre atividades cotidianas têm sido realizadas com base nos estudos de Marcadores de Estresse Ocupacional (MEO), termo que designa um conjunto de evidências no esqueleto, entre as quais marcadores não patológicos e patológicos, potencialmente capazes de informar acerca das solicitações mecânicas ocorridas ao longo da vida, principalmente aquelas relacionadas a atividades repetitivas e/ou intensas.

O princípio biomecânico, conhecido como Lei de Wolff, considera o osso um tecido dinâmico, o qual possui a capacidade de ajustar sua forma e distribuição em resposta às demandas externas. Dessa forma, a prática de atividades que demandam esforço físico regular e prolongado altera a morfologia óssea, estimulando a hipertrofia do tecido em uma tentativa de acomodar adequadamente cargas cada vez maiores (Knüsel, 2000). O mesmo princípio foi definido por Neves (1984), por meio do termo 'plasticidade', que é a capacidade dos componentes ósseos do esqueleto de responderem, dentro de certos limites, a estímulos internos ou externos ao corpo, oriundos da vida diária e das condições ambientais nas quais os indivíduos se encontram.

Entre os principais tipos de MEO, podemos citar as fraturas por estresse, os nódulos de Schmorl, os marcadores de estresse músculo esquelético, os marcadores de estresse mecânico postural e as osteoartroses (Merbs, 1983; Kennedy, 1989; Capasso et al., 1999), sendo estas últimas um dos processos patológicos mais observados e de diagnóstico menos controverso em esqueletos arqueológicos, daí sua importância para os estudos paleoepidemiológicos (Waldron, 1992; Weiss e Jurmain, 2007).

A associação entre osteoartrose e sobrecarga mecânica sobre as articulações tem sido demonstrada principalmente por meio de estudos epidemiológicos com atletas, cujas atividades físicas demandam esforço intenso e repetitivo (revidado por Lequesne et al., 1997; referências em Resnick e Niwayama, 1988, p. 1369). Estudos voltados especificamente para a associação entre osteoartrose e atividades ocupacionais, tais como mineração de carvão e trabalho braçal em estaleiros, também demonstram a expressiva contribuição das demandas mecânicas no comprometimento articular. Em relação a estresses mecânicos específicos, uma série de estudos de caso-controle demonstra, de forma consistente, que escaladas e posição ajoelhada, por exemplo, estão associadas ao desenvolvimento da osteoartrose. Correlações positivas entre atividades laborais e osteoartrose em articulações específicas também têm sido demonstradas. $\bigcirc$ comprometimento articular no quadril, por exemplo, foi associado a atividades ligadas à agricultura e ao carregamento de peso (revisado por Felson et al., 2000; Lievense et al., 2001).

Provavelmente devido à alta frequência em populações antigas, as osteoartroses têm chamado a atenção dos bioarqueólogos norte-americanos e europeus há décadas. A quantificação e interpretação dos dados segundo as variáveis sexo, idade e lateralidade têm dado suporte a inferências cada vez mais consistentes sobre divisão de tarefas por gênero ou por distintos segmentos sociais. Os padrões de comprometimento articular, estabelecidos a partir da distribuição das frequências de osteoartrose segundo superfície articular e assimetrias contralaterais, permitem a identificação dos membros mais solicitados e do tipo de movimento mais frequente e/ou intensamente realizado por esses membros. Quando associados aos contextos materiais e ambientais, esses dados possibilitam inferências mais específicas sobre aspectos da organização social diária, 
sendo possível a proposição das atividades realizadas, principalmente as associadas aos sistemas de subsistência, tais como os deslocamentos terrestres e aquáticos para busca e transporte de alimentos e matérias-primas, assim como sua preparação, entre outras.

Neste sentido, a comparação quantitativa e qualitativa de dados entre séries de caçadores-coletores e horticultores, ou entre grupos com maior ou menor mobilidade, tem revelado resultados particularmente interessantes no que se refere às diferenças associadas aos distintos estilos de vida dessas populações, principalmente quanto às estratégias adaptativas (por exemplo, Bridges, 1991; Lieverse et al., 2007).

\section{PROBLEMÁTICA: MÉTODOS DIAGNÓSTICOS PARA OSTEOARTROSE}

A osteoartrose é um processo patológico articular multifatorial. Embora os bioarqueólogos tenham se concentrado nos efeitos do estresse mecânico repetitivo e da idade como principais fatores responsáveis pelo seu desenvolvimento e severidade, estudos clínicos têm identificado outros fatores capazes de influenciar a osteoartrose, tais como a anatomia das estruturas, o peso corporal, os hormônios e a genética (Weiss e Jurmain, 2007). Ou seja, embora a degeneração articular represente o resultado da incidência de cargas repetitivas e/ou excessivas sobre a articulação, sua capacidade de resposta e resistência a estas cargas pode variar populacional e individualmente, devido aos fatores supracitados (Salter, 2002). Comparações quantitativas e qualitativas entre os sexos, entre distintas faixas etárias ou entre populações biologicamente não relacionadas devem, portanto, ser feitas com cautela.

Do ponto de vista clínico, a osteoartrose caracteriza-se principalmente por dor nas articulações afetadas, pela limitação de movimentos e crepitação. Histologicamente, a patologia é inicialmente caracterizada pela fragmentação da superfície da cartilagem, multiplicação dos condrócitos, fissuras verticais e remodelação (Brandt et al., 2008). Do ponto de vista patológico, a osteoartrose corresponde a áreas focalizadas de perda da cartilagem em articulações sinoviais, associadas à hipertrofia óssea (esclerose do osso subcondral e osteofitos), ao espessamento da cápsula articular e ao estreitamento do espaço articular (Kellgren e Lawrence, 1957). Estas alterações, que envolvem todos os tecidos do sistema articular, representam um critério diagnóstico padronizado e amplamente utilizado na clínica moderna, sendo observadas por meio de técnicas de imagem, principalmente a radiografia.

Em séries esqueléticas, a osteoartrose é observada de forma direta e apenas a partir de alterações ósseas nas superfícies articulares, logo, os critérios diagnósticos são distintos daqueles utilizados na clínica. Mesmo os marcadores associados às alterações ósseas apresentam variações quando observados nos indivíduos vivos por meio de técnicas de imagem, ou diretamente no osso. A falta de correspondência entre os marcadores e os sistemas radiográficos de escores para diagnóstico, amplamente aceitos e utilizados na clínica, e o conjunto de evidências observáveis diretamente no osso subcondral resultaram na aplicação de metodologias não padronizadas para diagnóstico em séries esqueléticas arqueológicas.

Dessa forma, apesar de a osteoartrose ser considerada na literatura especializada internacional uma patologia de fácil diagnóstico, uma revisão cuidadosa dos estudos paleoepidemiológicos e clínicos sobre o tema apontou uma série de problemas metodológicos relacionados ao estabelecimento dos marcadores, assim como ao seu registro, à quantificação e interpretação, o que pode comprometer expressivamente a qualidade e validade dos estudos sobre atividades cotidianas e estilos de vida. A falta de padronização metodológica observada nos estudos de osteoartrose (Tabela 1), por outro lado, inviabiliza comparações entre diferentes estudos, muitas vezes realizados com grupos cultural e/ou biologicamente afins. 
Tabela 1. Exemplos de metodologias utilizadas em estudos paleoepidemiológicos sobre osteoartrose. Legendas: $\mathrm{A}=$ áreas específicas da superfície articular; $\mathrm{SA}=$ superfície articular; $\mathrm{CA}=$ conjunto articular.

(Continua)

\begin{tabular}{|c|c|c|c|}
\hline Critério diagnóstico & $\begin{array}{l}\text { Unidade de } \\
\text { análise }\end{array}$ & $\begin{array}{l}\text { Atribuição de } \\
\text { graus de intensidade }\end{array}$ & Referência \\
\hline $\begin{array}{l}\text { Formação óssea hipertrófica na periferia e na superfície articular; } \\
\text { porosidade na superfície articular; eburnação }\end{array}$ & A & Não & Ortner (1968) \\
\hline $\begin{array}{c}\text { Na superfície articular: porosidade, eburnação, destruição; na } \\
\text { margem da superfície articular: osteófito; inserções ligamento- } \\
\text { tendinosas; osteófito epiarticular }\end{array}$ & CA & $\begin{array}{l}\text { Três unidades de } \\
\text { escala ordinal }\end{array}$ & Jurmain (1977) \\
\hline $\begin{array}{c}\text { Formação de múltiplas e pequenas cavidades (osteoporose) no } \\
\text { centro da superfície articular; neoformação óssea (exostose) na } \\
\text { margem da cartilagem articular, denominada osteófito; eburnação e } \\
\text { polimento }\end{array}$ & CA & $\begin{array}{l}\text { Quatro unidades de } \\
\text { escala ordinal }\end{array}$ & Neves (1984) \\
\hline Labiamento; porosidade; eburnação & $\begin{array}{c}\text { CA e A } \\
\text { (patela e úmero) }\end{array}$ & Sim & Bridges (1991) \\
\hline $\begin{array}{l}\text { Eburnação ou combinação dos seguintes marcadores: neoformação } \\
\text { óssea na margem ou na superfície articular; porosidade na superfície } \\
\text { articular; deformação do contorno normal da superfície articular }\end{array}$ & CA & Não & Waldron (1991) \\
\hline $\begin{array}{c}\text { Porosidade (áreas de pseudocisto); neoformação óssea nas margens } \\
\text { articulares (labiamento/osteófito); eburnação }\end{array}$ & SA e A & Sim & Merbs (1983) \\
\hline $\begin{array}{l}\text { Eburnação ou combinação dos seguintes marcadores: osteofitoses } \\
\text { nas margens articulares; porosidade na superfície articular }\end{array}$ & CA & Não & $\begin{array}{l}\text { Rogers e Dieppe } \\
\quad(1994)\end{array}$ \\
\hline Eburnação; usura; cavidades císticas; osteófitos & CA & Não & Souza (1995) \\
\hline Eburnação & CA & Não & Waldron (1997) \\
\hline $\begin{array}{l}\text { Reação óssea subcondral; osteófitos marginais; porosidade na } \\
\text { superfície articular; alteração do contorno da articulação }\end{array}$ & CA & Não & $\begin{array}{l}\text { Baetsen et al. } \\
\quad(1997)\end{array}$ \\
\hline $\begin{array}{l}\text { Eburnação, osteófitos marginais, porosidade na superfície articular; } \\
\text { alteração do contorno normal da superfície articular }\end{array}$ & A & Não & $\begin{array}{l}\text { Inoue et al. } \\
\text { (2001) }\end{array}$ \\
\hline $\begin{array}{l}\text { Porosidade, associada à presença de uma margem clara e afiada } \\
\text { em torno da face articular; labiamento (>1 mm); desgaste da face } \\
\text { articular; projeção óssea acentuada (>2 mm) }\end{array}$ & CA & $\begin{array}{l}\text { Quatro unidades de } \\
\text { escala ordinal }\end{array}$ & $\begin{array}{l}\text { Rodrigues- } \\
\text { Carvalho (2004) }\end{array}$ \\
\hline $\begin{array}{l}\text { Porosidade na superfície articular; labiamento na margem; } \\
\text { eburnação; osteófito }\end{array}$ & CA & $\begin{array}{l}\text { Três unidades de } \\
\text { escala ordinal }\end{array}$ & $\begin{array}{l}\text { Cope et al. } \\
\text { (2005) }\end{array}$ \\
\hline $\begin{array}{l}\text { Formação óssea periarticular; reabsorção do osso subcondral; } \\
\text { eburnação }\end{array}$ & A & Não & $\begin{array}{l}\text { Lieverse et al. } \\
\qquad(2007)\end{array}$ \\
\hline $\begin{array}{l}\text { Eburnação ou dois dos seguintes marcadores: neoformação óssea } \\
\text { ao redor da margem articular (labiamento); neoformação óssea na } \\
\text { superfície articular (osteófito); porosidade na superfície articular }\end{array}$ & CA & $\begin{array}{l}\text { Quatro unidades de } \\
\text { escala ordinal }\end{array}$ & Cardoso (2008) \\
\hline
\end{tabular}


Tabela 1.

(Conclusão)

\begin{tabular}{|c|c|c|c|}
\hline Critério diagnóstico & $\begin{array}{l}\text { Unidade de } \\
\text { análise }\end{array}$ & $\begin{array}{l}\text { Atribuição de } \\
\text { graus de intensidade }\end{array}$ & Referência \\
\hline Labiamento, osteófito, eburnação & A & Não & Estanek (2008) \\
\hline Labiamento, osteófito, porosidade, eburnação & - & $\begin{array}{l}\text { Quatro unidades de } \\
\text { escala ordinal }\end{array}$ & Robin (2008) \\
\hline Labiamento osteofiticoperiarticular & A & Não & $\begin{array}{l}\text { Klaus et al. } \\
\text { (2009) }\end{array}$ \\
\hline $\begin{array}{c}\text { Na ausência de eburnação, presença de outros dois marcadores: } \\
\text { osteófitos, porosidade, alteração da morfologia articular }\end{array}$ & CA & Não & Ponce (2010) \\
\hline Eburnação & - & Não & $\begin{array}{l}\text { Molnar et al. } \\
\qquad(2011)\end{array}$ \\
\hline Labiamento, porosidade, eburnação & CA & $\begin{array}{l}\text { Quatro unidades de } \\
\text { escala ordinal }\end{array}$ & $\begin{array}{l}\text { Woo e Sciulli } \\
\qquad(2013)\end{array}$ \\
\hline
\end{tabular}

A partir de um extenso levantamento das questões metodológicas que merecem revisão, está em desenvolvimento o projeto piloto "Novos aportes metodológicos para o diagnóstico e a interpretação de osteoartroses em séries esqueléticas arqueológicas", cujos objetivos são identificar, definir e caracterizar os marcadores ósseos de osteoartrose mais adequados para a análise em remanescentes arqueológicos, além de padronizar os métodos diagnósticos e de documentação, buscando-se, assim, aprofundar o nível das inferências sustentadas pelos padrões de comprometimento articular. Espera-se, por meio dessa iniciativa, beneficiar principalmente pesquisadores mais jovens, com pouca experiência no tema, além de incentivar o interesse pelos estudos de osteoartrose em séries brasileiras, desenvolvidos de forma embrionária até o momento, mas imbuídos de grande potencial para contribuir com o avanço da nossa arqueologia.

No presente artigo, será abordado um dos aspectos metodológicos desenvolvidos no projeto supracitado - o registro dos processos tafonômicos e dos marcadores de osteoartrose -, o qual representa uma importante etapa no processo de análise do comprometimento articular. Será apresentada também uma breve discussão sobre os estudos de osteoartrose já desenvolvidos e seu potencial informativo para a arqueologia brasileira.

\section{METODOLOGIA GERAL}

Até o momento, a pesquisa concentrou-se sobre as articulações sinoviais mais representativas em material arqueológico (Tabela 2), por estarem fortemente correlacionadas com o estresse mecânico resultante de movimentos repetitivos e/ou vigorosos dos membros. Os marcadores considerados foram labiamento, osteofitos,

Tabela 2. Número de conjuntos articulares analisados e respectiva localização das superfícies articulares. Legenda: $*$ não foi incluída a superfície articular da fíbula, uma vez que a articulação tíbio-fibular é uma sindesmose, com movimento extremamente reduzido.

\begin{tabular}{c|c|c}
\hline $\begin{array}{c}\text { Conjunto } \\
\text { articular }\end{array}$ & $\begin{array}{c}\text { Localização das } \\
\text { superfícies articulares }\end{array}$ & Total \\
\hline Ombro & Face glenoide; cabeça do úmero & 69 \\
\hline Cotovelo & $\begin{array}{c}\text { Epífise distal do úmero; epífises } \\
\text { proximais da ulna e do rádio }\end{array}$ & 78 \\
\hline Quadril & Acetábulo; cabeça do fêmur & 55 \\
\hline Joelho & $\begin{array}{r}\text { Patela; epífise distal do fêmur; epífise } \\
\text { distal da tíbia }\end{array}$ & 43 \\
\hline Tornozelo* & $\begin{array}{r}\text { Epífise distal da tíbia; superfície } \\
\text { proximal do tálus }\end{array}$ & 43 \\
\hline
\end{tabular}

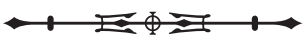


porosidade, neoformação óssea na superfície articular e eburnação e, quando possível, esclerose e cistos subcondrais. A validade desses marcadores para os estudos de comprometimento articular será discutida em outra publicação, uma vez que este tema foge ao escopo do presente trabalho.

As séries estudadas, provenientes dos sítios précoloniais litorâneos Praia da Tapera (Santa Catarina, Museu do Homem do Sambaqui), Piaçagüera (São Paulo, Museu de Arqueologia e Etnologia) e Tenório (São Paulo, Museu de Arqueologia e Etnologia), são compostas por indivíduos adultos de ambos os sexos.

Indivíduos que apresentaram quaisquer patologias ou condições que possam ter influência direta ou indireta sobre a osteoartrose, tais como fraturas, luxações, displasia e DISH, foram excluídos das séries examinadas. Para os propósitos do presente trabalho, foram considerados apenas os conjuntos articulares completos.

\section{COLETA DE DADOS: O REGISTRO DOS PROCESSOS TAFONÔMICOS E DOS MARCADORES DE OSTEOARTROSE}

Entre as dezenas de estudos publicados sobre osteoartrose, são raros aqueles que apresentam os desenhos esquemáticos das superfícies articulares utilizados nas fichas de coleta de dados. Estes esquemas constituem-se em importante instrumento de documentação dos marcadores, principalmente no que se refere à área articular afetada, extensão da lesão e extensão das áreas ausentes devido a processos tafonômicos, especialmente as quebras. São exceções o manual "Standards for data collection from human skeletal remains" (Buikstra e Ubelaker, 1994), amplamente referido na literatura nacional e internacional, além de raras dissertações e teses (por exemplo, Estanek, 2008), as quais, no entanto, têm circulação restrita.

A utilização, pela autora, destes esquemas disponíveis, demonstrou inadequação, em maior ou menor grau, para a documentação dos marcadores de osteoartrose, seja pelo fato de não terem sido elaborados especificamente para este fim, ou ainda porque não contemplam todas as áreas articulares efetivamente passíveis de serem afetadas por osteoartrose. Em alguns casos, embora as áreas envolvidas estejam representadas, a simplificação dos acidentes anatômicos também prejudica o registro exato dos marcadores. A fim de suprir essas lacunas, muitas vezes é necessária a realização de desenhos adicionais nas fichas ou, ainda, uma descrição detalhada da área afetada, tornando o trabalho mais lento e, algumas vezes, o registro impreciso.

A extensão das áreas articulares ausentes devido a processos tafonômicos é outra questão relevante. Tal como ocorre em relação aos marcadores, a utilização de esquemas inadequados prejudica o registro das áreas ausentes/presentes. A revisão da literatura demonstrou que, na realidade, este registro sequer tem sido realizado, tampouco sendo discutidas as consequências dos processos tafonômicos sobre a quantificação dos distintos marcadores. $\bigcirc$ estado de preservação das articulações, no entanto, é, ou deveria ser, um dos critérios para eleição das articulações adequadas para análise. Os poucos trabalhos que explicitam o método utilizado (por exemplo, Cardoso, 2008; Estanek, 2008) apresentam como critério a simples presença de pelo menos 50\% de sua superfície articular. Mas qual 50\% foi examinado? $O$ que esta área representa em termos biomecânicos? A ausência de discussões sobre estes aspectos está subjacente à ideia de que as superfícies articulares, e mesmo o conjunto articular, representam uma unidade homogênea.

O estresse mecânico, no entanto, pode impactar de forma mais ou menos intensa as diferentes áreas da superfície articular em função das áreas de contato intra-articulares, definidas a partir da sua congruência e dos movimentos realizados (Frost, 1999). Dessa forma, a ausência dos marcadores em apenas parte da superfície articular, ou seja, a parte presente, não quer dizer absolutamente ausência de osteoartrose, uma 
vez que a exposição ao risco é diferenciada entre suas diferentes áreas.

Estudos específicos sobre determinação de áreas de contato articular identificam, com precisão, diferenças não apenas na localização, mas também no tamanho destas áreas, em função do tipo e da amplitude dos movimentos. Na articulação gleno-umeral, por exemplo, com o aumento da elevação do braço em rotação externa, a área de contato na cabeça do úmero migra da região inferior (89\% dos casos, em $0^{\circ}$ de elevação) para a região superocentral-posterior ( $84 \%$ dos casos em elevação total do braço, em $180^{\circ}$ ). Da mesma forma, uma migração em sentido posterior na área de contato da glenoide também pode ser observada (aumento de $33 \%$ dos casos, em $0^{\circ}$ de elevação, para 57\%, em $180^{\circ}$ ) (Soslowsky et al., 1992).

Mesmo em articulações com grau 1 de liberdade de movimento, ou uniaxiais (como a articulação fêmurpatelar), os marcadores podem estar localizados em áreas específicas da superfície articular, em função da extensão dos movimentos. Um bom exemplo é a patela, mapeada por Goodfellow e colaboradores (1976), cujas áreas de contato com o fêmur foram definidas na região superior, quando em extensão; na região central, quando em flexão de $45^{\circ}$; na região inferior, quando em flexão de $20^{\circ}$; e nas regiões medial e lateral, quando em $135^{\circ}$ ou flexão máxima.

Uma tentativa de mapear os locais específicos de ocorrência de marcadores foi realizada por Estanek (2008), a partir da divisão das superfícies articulares em quadrantes. O potencial representado por esta metodologia, até então inédita para o exame de articulações sinoviais em séries brasileiras, reside na possibilidade de serem localizados os marcadores e identificada sua extensão de forma mais exata. Os quadrantes propostos em alguns dos esquemas, no entanto, não representam efetivamente as áreas de contato específicas mais impactadas pela incidência de forças sobre a articulação. A definição dos quadrantes, tendo como base estudos específicos sobre movimento x congruência articular x estresse mecânico, forneceria uma ferramenta bastante útil para uma identificação mais precisa das demandas mecânicas sobre as articulações.

Uma quantificação exata das áreas ausentes/ presentes em cada conjunto articular permite também, consequentemente, a quantificação individualizada das articulações que os compõem. É o caso da articulação do cotovelo, a qual, apenas na porção distal do úmero, é composta pelas articulações úmero-ulnar e úmero-radial. Além de diferenças na incidência de força em função da amplitude dos movimentos de flexão e extensão, ocorrem diferenças também em função do tipo de movimento realizado - flexão/extensão e pronação/supinação, o que se reflete diretamente na ocorrência dos marcadores.

Por outro lado, independentemente da congruência das superfícies articulares e dos movimentos realizados, os diferentes marcadores de osteoartrose, devido à sua etiologia, se desenvolvem em regiões específicas da superfície articular. Os marcadores labiamento e osteófito, por exemplo, estão restritos às bordas da superfície articular. A questão a ser considerada é que esta é uma região particularmente susceptível a processos tafonômicos. Pouco protegidas pela fina camada de osso subcondral e constantemente impactadas durante o manuseio do osso ou devido a um acondicionamento inadequado, é comum a observação de bordas destruídas mesmo em ossos cujas demais estruturas articulares encontram-se íntegras. Nestes casos, a superfície articular tem sido simplesmente quantificada para todos os marcadores em questão.

A fragilidade das bordas das articulações, portanto, acaba por provocar um viés metodológico, subestimando as frequências para os marcadores supracitados, os quais, dependendo da extensão de destruição destas regiões, podem ser considerados inadequados para um determinado conjunto articular ou mesmo para uma série específica. As mesmas articulações excluídas para a quantificação de labiamentos e osteófitos, porém, podem ser adequadas para a quantificação dos demais 
marcadores localizados nas áreas internas das superfícies articulares (Figura 1). Desta forma, sugere-se a quantificação dos marcadores separadamente, segundo localização (bordas $x$ área interna da superfície articular), garantindo assim que os denominadores representem efetivamente o número de superfícies articulares com as áreas associadas presentes. A utilização desta metodologia deve minimizar erros sistemáticos na quantificação dos dados, e consequentemente na interpretação dos resultados.

A partir dos exemplos aqui discutidos, percebe-se a importância de que o registro das áreas presentes/ausentes seja o mais exato possível, assim como dos marcadores de osteoartrose, os quais primeiramente devem ser quantificados a partir de cada superfície articular e área específica, a fim de que os movimentos a eles relacionados sejam inferidos. A agregação dos dados a partir da região anatômica (por exemplo, epífise distal do úmero) ou do conjunto articular, embora seja adequada para uma visão mais geral do comprometimento articular em uma série esquelética, dificulta uma abordagem mais complexa e informativa em termos de demandas físicas e estilo de vida.

É certo que a utilização dos métodos acima discutidos, embora seja relevante, torna o registro e a análise do comprometimento articular ainda mais lentos e complexos. Salienta-se novamente, no entanto, que o uso de esquemas adequados, os quais contemplem efetivamente todas as áreas sujeitas à ocorrência dos distintos marcadores, facilita em grande medida os registros mencionados.

A partir desta perspectiva, foram elaborados desenhos esquemáticos das superfícies articulares examinadas, em todas as normas anatômicas adequadas para o registro dos marcadores (Figuras 2, 3 e 4). As áreas/ normas anatômicas representadas foram definidas a partir da coerência biomecânica dos movimentos permitidos em cada conjunto articular (planos sagital, frontal e transversal), os quais são regulados pela morfologia da articulação e pelo grau de liberdade dos movimentos (Tabela 3). Além de padronizar o registro dos marcadores, a utilização destes desenhos torna mais acurado e rápido o processo de documentação, sem que sejam necessárias descrições extensas da localização exata dos marcadores e dos movimentos a eles associados.

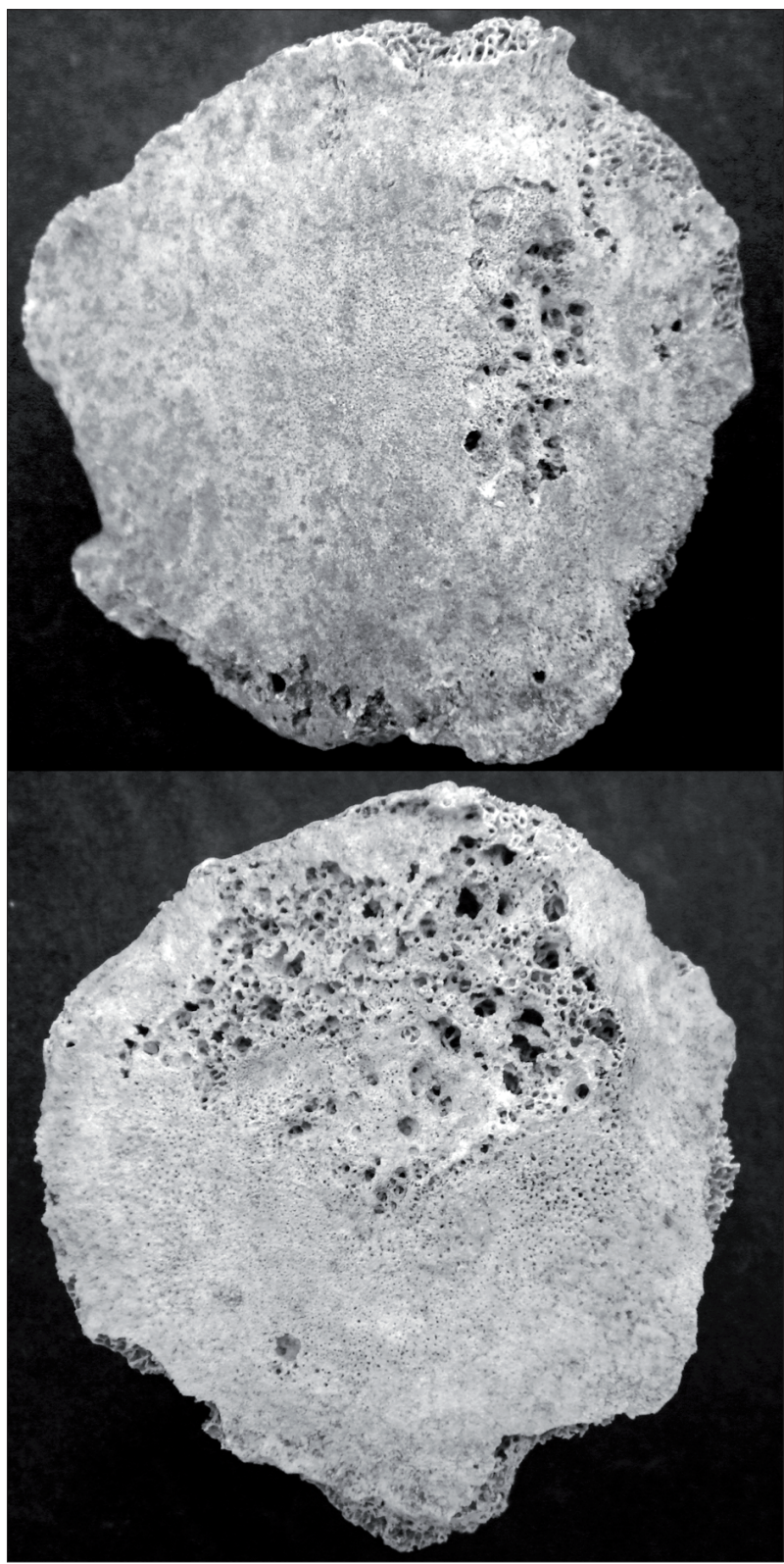

Figura 1. Patela esquerda. Note-se que houve quebra tafonômica em praticamente toda a borda, tornando-a inadequada para exame de labiamento e osteófitos. A área interna da articulação, no entanto, permanece preservada, apresentando porosidade e pontos de neoformação óssea na faceta medial. Fotos: A. Lessa.

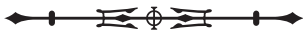




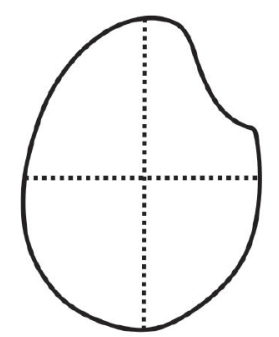

Glenóide D vista lateral

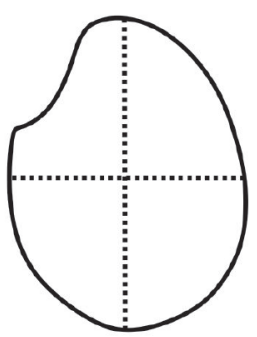

Glenóide E vista lateral

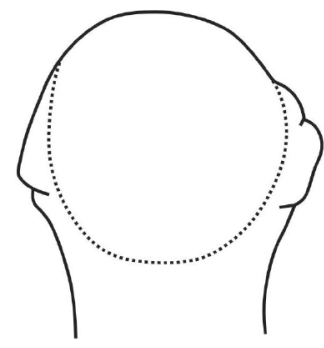

Úmero proximal D vista medial

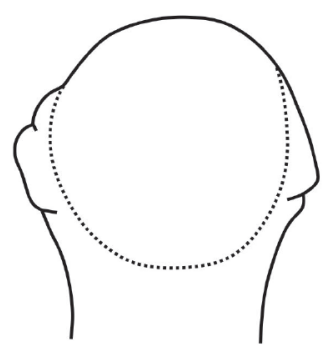

Úmero proximal E vista medial

\section{SUPERFÍCIES ARTICULARES DO QUADRIL}

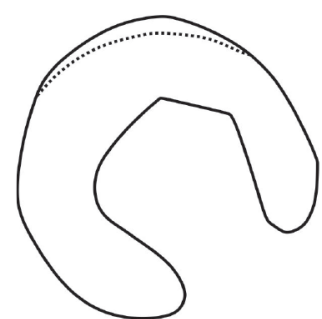

Acetábulo D vista lateral

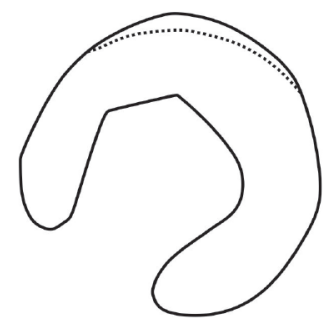

Acetábulo E vista lateral

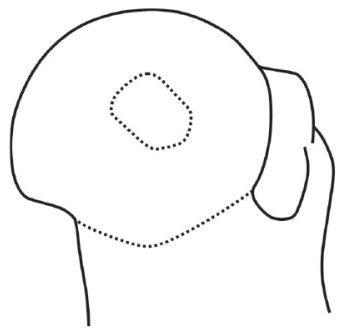

Fêmur proximal D vista medial

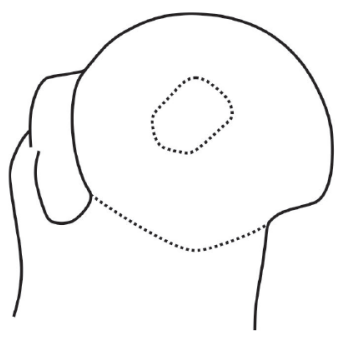

Fêmur proximal E vista medial

\section{SUPERFÍCIES ARTICULARES DO TORNOZELO}

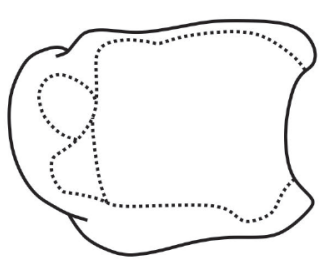

Tíbia distal D vista inferior

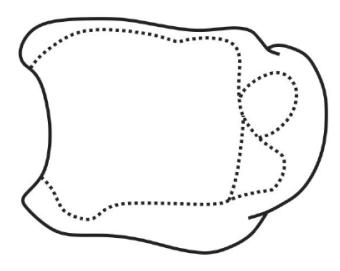

Tíbia distal E vista inferior

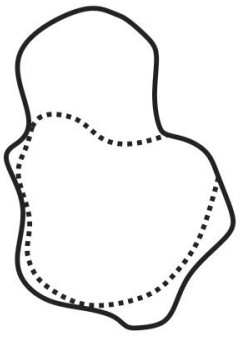

Tálus D vista dorsal

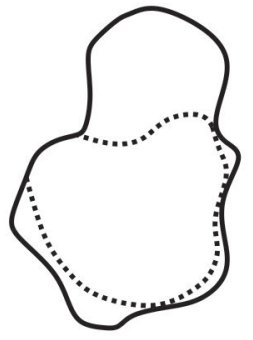

Tálus E vista dorsal

Figura 2. Esquema das superfícies articulares do ombro, quadril e tornozelo. Desenho: L. Witowski.

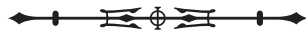




\section{SUPERFÍCIES ARTICULARES DO COTOVELO}

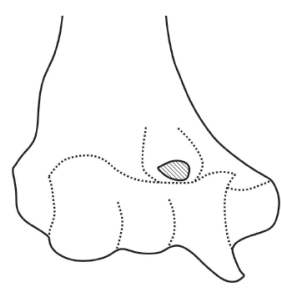

Úmero distal D vista anterior

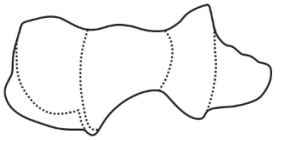

Úmero distal D vista inferior

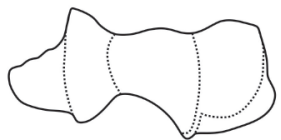

Úmero distal E vista inferior

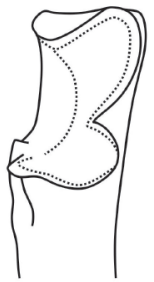

Ulna proximal D vista anterior

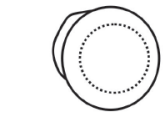

Rádio proximal D vista superior

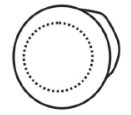

Rádio proximal E vista superior

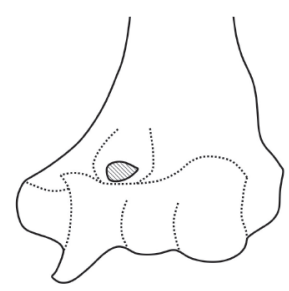

Úmero distal E vista anterior
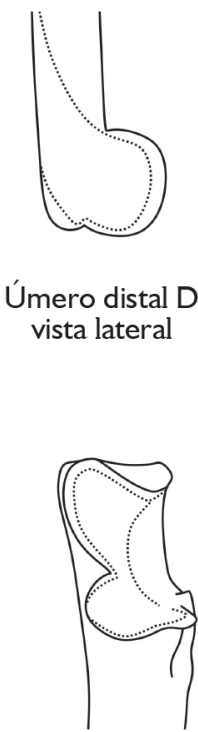

Ulna proximal E vista anterior

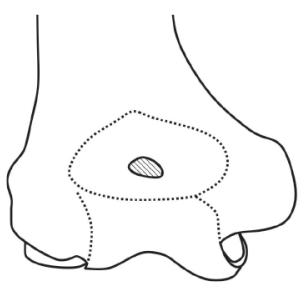

Úmero distal E vista posterior

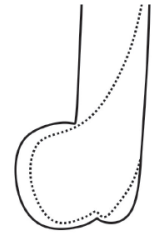

Úmero distal E
vista lateral

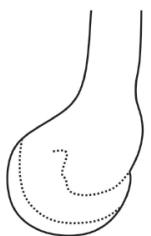

Úmero distal D vista medial

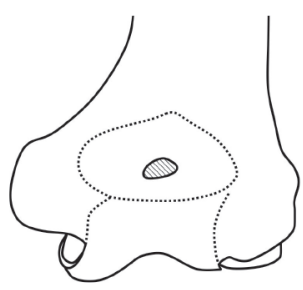

Úmero distal D vista posterior

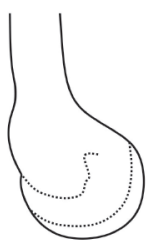
Úmero distal E
vista medial

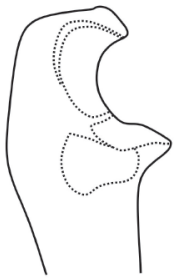

Ulna proximal D vista lateral

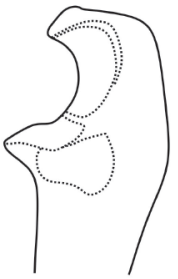

Ulna proximal E vista lateral

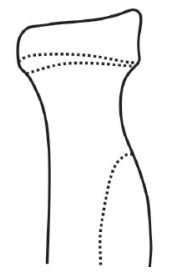

Rádio proximal D vista anterior

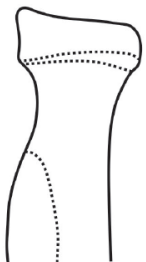

Rádio proximal E vista anterior

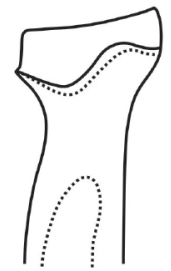

Rádio proximal $D$ vista medial

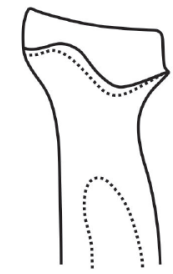

Rádio proximal E vista medial

Figura 3. Esquema das superfícies articulares do cotovelo. Desenho: L. Witowski.

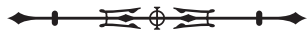




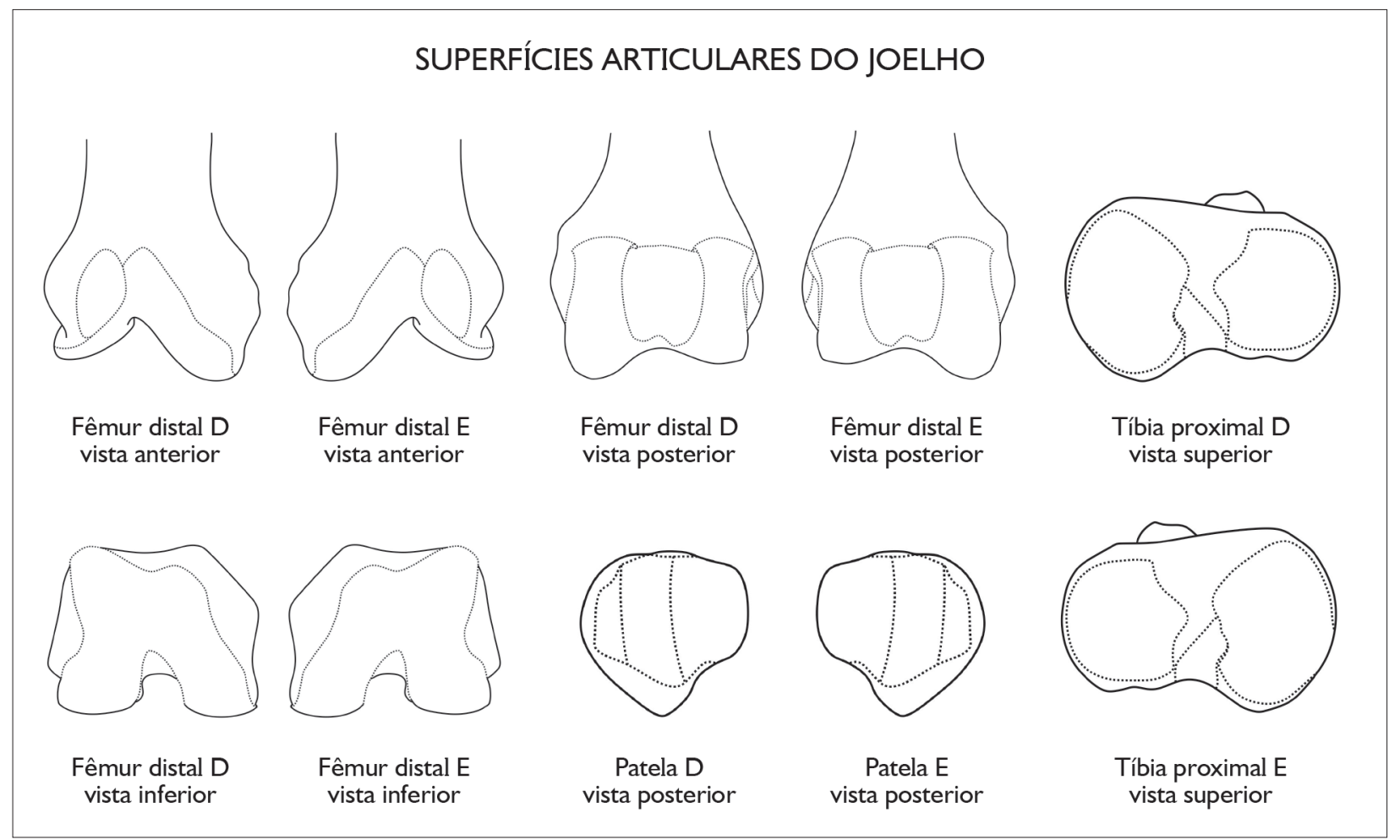

Figura 4. Esquema das superfícies articulares do joelho. Desenho: L. Witowski.

Tabela 3. Critérios utilizados para elaboração dos desenhos esquemáticos das superfícies articulares examinadas.

\begin{tabular}{|c|c|c|c|c|c|}
\hline $\begin{array}{l}\text { Região } \\
\text { anatômica }\end{array}$ & Articulação & Tipo & Plano & Movimento & $\begin{array}{l}\text { Grau de } \\
\text { liberdade }\end{array}$ \\
\hline \multirow{5}{*}{ Ombro } & \multirow{5}{*}{ Escápulo-umeral } & \multirow{5}{*}{ Esferoidal } & Sagital & Flexão/extensão/hiperextensão do braço & \multirow{5}{*}{ Triaxial } \\
\hline & & & Frontal & Adução/abdução lateral do braço & \\
\hline & & & Transversal & Adução/abdução horizontal do braço & \\
\hline & & & Sagital & Rotação interna/externa do braço & \\
\hline & & & - & Circundução do braço & \\
\hline \multirow{3}{*}{ Cotovelo } & Úmero-ulnar & Gínglimo & Sagital & Flexão/extensão do antebraço & \multirow[b]{2}{*}{ Uniaxial } \\
\hline & Úmero-radial & Gínglimo & Sagital & $\begin{array}{c}\text { Flexão/extensão e pronação/supinação do } \\
\text { antebraço }\end{array}$ & \\
\hline & Rádio-ulnar & Trocóidea & Transversal & Pronação/supinação do antebraço & Uniaxial \\
\hline \multirow{3}{*}{ Quadril } & \multirow{3}{*}{ Coxo-femural } & \multirow{3}{*}{ Esferoidal } & Sagital & Flexão/extensão/hiperextensão da coxa & \multirow{3}{*}{ Triaxial } \\
\hline & & & Frontal & Adução/abdução da coxa & \\
\hline & & & Transversal & Adução/abdução horizontal da coxa & \\
\hline \multirow{2}{*}{ Joelho } & Fêmur-tibial & Condilar & Sagital & Flexão/extensão da perna & Biaxial \\
\hline & Fêmur-patelar & Plana & Sagital & Flexão/extensão da perna & Uniaxial \\
\hline Tornozelo & Talo-crural & Gínglimo & Sagital & Dorsiflexão/flexão plantar do pé & Uniaxial \\
\hline
\end{tabular}




\section{IMPORTÂNCIA DOS ESTUDOS DE OSTEOARTROSE E OUTROS MEO PARA A ARQUEOLOGIA BRASILEIRA}

No Brasil, os poucos estudos específicos sobre osteoartrose, focalizando principalmente grupos sambaquieiros das regiões sul e sudeste, têm apresentado interessantes resultados no que se refere às atividades cotidianas e ao estilo de vida (Machado, 1984; Neves, 1984; Souza, 1995; Rodrigues-Carvalho, 2004; Estanek, 2008). Embora ainda timidamente, os dados sobre osteoartrose vêm contribuindo para as discussões que colocaram em xeque o modelo tradicional para grupos pré-coloniais que ocuparam a costa brasileira, o qual consiste em uma 'versão litorânea' do conceito de grupo caçador-coletor clássico, com ênfase na alta mobilidade, no entendimento do molusco como principal alimento e do sambaqui como assentamento temporário, resultado da acumulação de restos alimentares (Rodrigues-Carvalho, 2004).

Os paradigmas atuais propõem uma ocupação litorânea com estruturação sociocultural complexa, sendo postulada a pesca como principal meio de subsistência (Figuti, 1993; Figuti e Klokler, 1996); a ocupação de assentamentos permanentes e com hierarquização em relação aos sambaquis próximos (Lima, 1999-2000; Gaspar, 2003); o desenvolvimento de comunidades com densidade populacional expressiva (Fish et al., 1998; Gaspar, 2003); o desenvolvimento de um sistema de exploração conjunta do ambiente, com base na sobreposição de territórios explorados, o que minimizaria os custos de circulação de recursos e informação entre grupos que habitavam distintos assentamentos (Gaspar, 2000a, 2003); e a existência de um sistema social complexo de compartilhamento de princípios e costumes, o qual teria atuado como um eficiente regulador das tensões sociais (Lessa e Gaspar, no prelo).

Entre os principais resultados dos estudos de osteoartrose, as baixas frequências de comprometimento articular nos membros inferiores, observadas em todas as séries estudadas, reforçam a ideia de baixa mobilidade terrestre cotidiana (Machado, 1984; Neves, 1984; Souza, 1995; Rodrigues-Carvalho, 2004; Okumura e Eggers, 2005; Petronilho, 2005; Estanek, 2008). Frequências mais altas ou exclusivas entre homens, por sua vez, corroboram o padrão clássico de realização de tarefas que exijam deslocamentos constantes mais distantes do núcleo habitacional pela população masculina (Neves, 1984; Souza, 1995; Rodrigues-Carvalho, 2004; Estanek, 2008).

Por outro lado, os padrões de lesão observados para os membros superiores dão suporte ao uso de remos (Neves, 1984; Estanek, 2008), cuja implicação relaciona-se não apenas ao padrão de mobilidade, mas também às estratégias de subsistência, no caso, a pesca próxima à costa e em alto mar.

Os poucos dados para osteoartrose obtidos até o momento sugerem também estilos de vida diferenciados entre grupos litorâneos no que se refere a questões de gênero e do papel socioeconômico das mulheres. Embora tradicionalmente seja esperado que os homens estejam envolvidos com as atividades que demandam maior esforço físico e, consequentemente, apresentem comprometimento articular mais intenso, algumas exceções foram observadas. É o caso da série de pescadores-coletores do sítio Corondó, no Rio de Janeiro, na qual foram observadas frequências gerais mais altas entre as mulheres (Machado, 1984), e da série do sambaqui Ilhote do Leste, Rio de Janeiro, cujas frequências médias são mais altas do que aquelas observadas para os indivíduos masculinos de outras séries do Rio de Janeiro (Rodrigues-Carvalho, 2004).

A observação de indivíduos de mesmo sexo com diferentes graus de comprometimento articular dentro da mesma faixa etária em séries do litoral fluminense sugere diferenças de atividades ou demandas. Esse fato pode ser explicado principalmente pelo desempenho de tarefas ou papéis sociais específicos, em função de condições pessoais diferenciadas, tanto do ponto de vista biológico quanto sociocultural. Já o padrão precoce de sinais leves e moderados de osteoartrose, além de indicar a inclusão 
de indivíduos muito jovens em práticas cotidianas que demandam esforço físico acentuado (Rodrigues-Carvalho, 2004), sugere uma etiologia efetivamente mecânica para o surgimento e desenvolvimento das osteoartroses, independentemente do fator idade, o que chama a atenção para a necessidade de cautela ao se comparar populações antigas e modernas.

Outra questão de vital importância para a arqueologia do litoral brasileiro, apontada por Gaspar (2003) e problematizada por Rodrigues-Carvalho (2004), diz respeito à tendência em uniformizar em excesso as interpretações sobre os estilos de vida desses grupos pescadores-coletores, na medida em que ainda se trabalha uma categoria genérica como a de 'sambaqui', baseada em características morfo-constitucionais gerais dos sítios arqueológicos, e que acaba por guiar a construção de outra categoria genérica, a de 'grupos sambaquieiros'.

De uma forma geral, esses grupos, cuja distribuição geográfica demonstra uma ocupação expressiva e sistemática desde o sul de Santa Catarina até pelo menos o norte do Rio de Janeiro (não obstante a presença de ocupações mais pontuais na Bahia, no Maranhão e no Pará), têm sido entendidos e classificados apenas de acordo com sua estratégia econômica predominante, a pesca e a coleta de moluscos, e, grosso modo, com base nos dados produzidos para os sítios médios, grandes e monumentais da área nuclear de Santa Catarina.

De fato, um olhar mais amplo sobre as evidências arqueológicas é capaz de reconhecer uma identidade social compartilhada, representada pela íntima associação entre os construtores de montes de conchas, os mortos e os restos alimentares e industriais (Gaspar, 2000b), assim como uma unidade ideológica, representada pela presença de zoólitos, desde o Uruguai até São Paulo (Prous, 1992). Essa perspectiva, no entanto, não deve inibir um foco mais direcionado para as particularidades que naturalmente permeiam qualquer sistema sociocultural.

Pesquisas arqueológicas, e mesmo bioarqueológicas, vêm evidenciando variações espaço-temporais em alguns aspectos da cultura material, das práticas funerárias e alimentares e dos padrões de saúde (por exemplo, Gaspar e DeBlasis, 1992; Kneip, 1994; Kneip e Machado, 1993; Souza, 1995; Wesolowski, 2000). Embora esses estudos apontem para a necessidade de se entender estes grupos sob uma perspectiva que contemple a diversidade e complexidade sociocultural, ainda são escassos os esforços sistemáticos para o desenvolvimento de abordagens mais integrativas dos dados arqueológicos e bioarqueológicos, buscando-se investigar tais variações enquanto parte das escolhas próprias de cada grupo.

A identificação e o entendimento dessa variabilidade é condição fundamental para a extrapolação dos quadros gerais sobre a ocupação pré-colonial da costa brasileira, a partir da construção de sínteses regionais, que possam contemplar também as particularidades próprias de cada estilo de vida. Nesse aspecto, o estudo das osteoartroses e demais MEO já começa a demonstrar seu potencial informativo.

Citando novamente o trabalho de RodriguesCarvalho (2004), foram observados padrões de lesão diferenciados nas séries provenientes dos sítios localizados no recôncavo de Saquarema, se comparados com os padrões observados para outras séries litorâneas. Esta variação, representativa de custos biológicos distintos, pode estar relacionada ao estabelecimento desses sítios em áreas próximas à lagoa de Saquarema, o que sugere uma preferência pela exploração do rico ambiente lagunar, em detrimento do ambiente marinho, caracterizado, naquela região, pela formação de grandes ondas. $\bigcirc$ estudo dos traumas agudos (Lessa, 2011) também aponta para uma diversidade biocultural no que se refere aos acidentes entre os indivíduos dessas séries, o que mais uma vez estaria em consonância com a exploração do ambiente lagunar. Embora sua representatividade numérica confira um caráter preliminar a ambos os estudos, já se delineia um diferenciado padrão de atividades cotidianas para grupos que ocuparam áreas próximas à lagoa de Saquarema. 
Ainda em relação aos estudos de MEO em séries do litoral fluminense, o trabalho de Lessa e Coelho (2010) demonstra diferenças adaptativas expressivas entre dois grupos sambaquieiros, provenientes dos sítios llhote do Leste e Zé Espinho. $O$ exame das espondilólises e dos nódulos de Schmorl demonstrou que o primeiro grupo mantinha um padrão de atividades cotidianas muito intensas, possivelmente associadas ao uso de embarcações em mar aberto; enquanto o segundo grupo esteve menos exposto a riscos de lesão, o que pode estar associado a uma intensiva exploração do ambiente de mangue e de uma baía de águas calmas. Dados arqueológicos, ambientais e bioarqueológicos dão suporte a esta interpretação, a qual enfatiza a necessidade de se entender o estilo de vida dos grupos sambaquieiros a partir de suas singularidades.

Os estudos supracitados sugerem, ainda de forma embrionária, a existência de estilos de vida diferenciados no litoral fluminense, organizados em função de uma exploração mais intensa de diferentes tipos de ambiente: as águas calmas de lagoas e baías e as águas agitadas do oceano. A ampliação dos estudos de osteoartrose em outras séries afins, com o uso de metodologia padronizada, constitui-se em uma ótima ferramenta para o aprofundamento dessa discussão, a partir dos dados sobre a intensidade e o tipo de comprometimento articular observado nesses grupos.

A uniformização observada acerca da construção do conhecimento sobre o estilo de vida litorâneo em período pré-colonial, no entanto, não se restringe aos grupos sambaquieiros, mas acaba por englobar outros grupos. É o caso dos pescadores-caçadores que ocuparam os denominados sítios rasos cerâmicos e pré-cerâmicos, muitos dos quais escavados por Rohr $(1959,1966)$ e Beck (1972, por exemplo) entre as décadas de 1950 e 1970. Evidências associadas a esses assentamentos são comuns principalmente na região sul do Brasil, embora em quantidade menor do que aquelas associadas a sambaquis, possivelmente em função da relativa dificuldade para localização/preservação dos sítios, cujas camadas arqueológicas têm, em média, 1,50 m. Ainda assim, as séries provenientes dos sítios de pescadores-caçadores estão entre as mais representativas do litoral, tanto do ponto de vista qualitativo quanto quantitativo, sendo um dos melhores exemplos a série Praia da Tapera (Florianópolis, Santa Catarina), que conta com 172 indivíduos em estado de preservação de regular a ótimo.

Apesar das diferenças ideológicas e sociais associadas à implementação dos dois tipos de assentamentos litorâneos supracitados, cujos resultados mais impactantes, a um primeiro olhar, estão na escolha ou não da construção dos montes de conchas, são raros até o momento os estudos arqueológicos ou bioarqueológicos sistemáticos para a caracterização de sua variabilidade cultural.

Uma primeira abordagem nesse sentido foi realizada por Lessa (2005) e Lessa e Scherer (2008), com base em questões relacionadas à conduta agressiva entre estes grupos, cuja frequência aumenta a partir, aproximadamente, do primeiro milênio da era cristã, período que coincide com o colapso do modo de vida sambaquieiro e a implementação dos assentamentos de pescadores-caçadores ceramistas. Foram propostas interpretações gerais e particulares não excludentes para o fenômeno, incluindo o surgimento de uma nova ordem, segundo a qual já não importavam as premissas associadas à acumulação de conchas; a introdução de uma importante inovação tecnológica, como a cerâmica; ou ainda uma mudança no padrão de subsistência, passando de uma exploração intensa de frutos do mar em geral e escassez de fauna terrestre, entre os grupos sambaquieiros, para uma maior diversificação alimentar entre pescadores-caçadores, o que inclui, além da pesca, a caça de um grande número de mamíferos terrestres e aquáticos.

\section{CONSIDERAÇÕES FINAIS}

As perspectivas acima mencionadas apontam para a necessidade e possibilidade de um olhar que contemporize aspectos particulares do estilo de vida de populações agrupadas em categorias muito amplas, como grupos

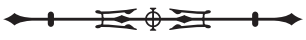


litorâneos ou grupos sambaquieiros. É preciso ter em mente que, embora tenham ocupado ambientes ecologicamente semelhantes, e tenham inclusive compartilhado uma mesma ideologia construtiva, no caso dos grupos sambaquieiros, sempre teremos que lidar com variações nos seus sistemas adaptativos. Essas variações, entendidas como ajustes particulares de cada sociedade, em função de sua bagagem cultural e das oportunidades e limitações impostas pelo meio, são a chave para a construção de um passado no qual o homem é o principal agente nas relações dentro do seu grupo, com seus aliados e inimigos, e com o ambiente no qual ele viveu. Nesse sentido, um impulso nos estudos de osteoartrose certamente contribuirá com dados mais específicos e acurados sobre o cotidiano destas populações.

\section{REFERÊNCIAS}

BAETSEN, S.; BITTER, P.; BRUINTJES, T. Hip and knee osteoarthritis in an Eighteenth Century urban population. International Journal of Osteoarchaeology, v. 7, n. 6, p. 628-630, 1997.

BECK, Ana Maria. A variação do conteúdo cultural dos sambaquis: litoral de Santa Catarina. 1972. Tese (Doutorado em Antropologia) - Universidade de São Paulo, São Paulo, 1972.

BRANDT, Kenneth; DIEPPE, Paul; RADIN, Eric. Etiopathogenesis of osteoarthritis. Rheumatic Disease Clinics of North America, $v$. 34, n. 3, p. 531-559, 2008.

BRIDGES, Patricia. Degenerative joint disease in hunter-gatherers and agriculturalists from the Southeastern United States. American Journal of Physical Anthropology, v. 85, n. 4, p. 379-391, 1991.

BUIKSTRA, Jane; UBELAKER, Douglas. Standards for data collection from human skeletal remains. Fayetteville: Arkansas Archaeological Survey, 1994.

CAPASSO, Luigi; KENNEDY, Kenneth; WILCZAK, Cynthia. Atlas of occupational markers on human remains. Teramo: Edigrafital, 1999.

CARDOSO, Francisca Alves. A portrait of gender in two 19th and 20th Century Portuguese populations: a paleopathological perspective. 2008. Tese (Doutorado em Antropologia Biológica) Durham University, Durham, 2008.

COPE, Janet; BERRYMAN, Allison; MARTIN, Debra; POTTS, Daniel. Robusticity and osteoarthritis at the trapeziometacarpal joint in a Bronze Age population from Tell Abraq, United Arab Emirates. American Journal of Physical Anthropology, v. 126, n. 4, p. 391-400, 2005.
ESTANEK, Angélica. Trabalho e convívio no paraíso insular: paleopatologia dos remanescentes ósseos do sítio llhote do Leste/RJ. 2008. Dissertação (Mestrado em Saúde Pública) - Escola Nacional de Saúde Pública Sérgio Arouca/Fundação Oswaldo Cruz, Rio de Janeiro, 2008.

FELSON, D.; LAWRENCE, R. C.; DIEPPE, P. A.; HIRSCH, R.; HELMICK, C. G.; JORDAN, J. M.; KINGTON, R. S.; LANE, N. E.; NEVITT, M. C.; ZHANG, Y.; SOWERS, M.; MCALINDON, T.; SPECTOR, T. D.; POOLE, A. R.; YANOVSKI, S. Z.; ATESHIAN, G.; SHARMA, L.; BUCKWALTER, J. A.; BRANDT, K. D.; FRIES, J. F. Osteoarthritis: new insights. Part I: the disease and its risk factors. Annals of Internal Medicine, v. 133, n. 8, p. 635-646, 2000.

FIGUTI, Levi. O homem pré-histórico, o molusco e o sambaqui: considerações sobre a subsistência dos povos sambaquieiros. Revista do Museu de Arqueologia e Etnologia, v. 3, p. 67-80, 1993.

FIGUTI, Levi; KLOKLER, Daniela. Resultados preliminares dos vestígios zooarqueológicos do sambaqui Espinheiros II (Joinville, SC). Revista do Museu de Arqueologia e Etnologia, v. 6, p. 169-187, 1996.

FISH, Suzane; DEBLASIS, Paulo; GASPAR, Maria Dulce; FISH, Paul. Incremental events in the construction of sambaquis, Southeastern Santa Catarina. In: REUNIÃO CIENTÍFICA DA SOCIEDADE DE ARQUEOLOGIA BRASILEIRA, 9., 1998, Rio de Janeiro. Anais... Rio de Janeiro: SAB, 1998.

FROST, Harold. Joint anatomy, design, and arthroses: insights of the Utah Paradigm. The Anatomical Record, v. 255, n. 2, p. 162 174, 1999.

GASPAR, Maria Dulce. Aspectos da organização social de pescadores-coletores: região compreendida entre a Ilha Grande e o delta do Paraíba do Sul, Rio de Janeiro. Pesquisas, série Antropologia, v. 59, p. 1-163, 2003.

GASPAR, Maria Dulce. Sambaqui: arqueologia do litoral brasileiro. Rio de Janeiro: Jorge Zahar, 2000a.

GASPAR, Maria Dulce. Zoólitos, peixes e moluscos: cultura material e identidade social. Encontros e Estudos, Brasília, v. 3, p. 13-23, 2000 b.

GASPAR, Maria Dulce; DEBLASIS, Paulo. Construção de sambaqui. In: REUNIÃO CIENTÍFICA DA SOCIEDADE DE ARQUEOLOGIA BRASILEIRA, 6., 1992, Rio de Janeiro. Anais... Rio de Janeiro: SAB, 1992. v. 1, p. 172-179.

GOODFELLOW, J.; HUNGERFORD, D. S.; ZINDEL, M. Patellofemoral joint mechanics and pathology - I. Functional anatomy of the patello-femoral joint. Journal of Bone and Joint Surgery, v. 58-B, p. 287-290, 1976.

INOUE, K.; HUKUDA, P.; FARDELLON, P.; YANG, Z.; NAKAI, M.; KATAYAMA, K.; USHIYAMA, T.; SARUHASHI, J.; HUANG, J.; MAYEDA, A.; CATTEDDU, I.; OBRY, C. Prevalence of large joint osteoarthritis in Asian and Caucasian skeletal populations. Rheumatology, v. 40, n. 1, p. 70-73, 2001.

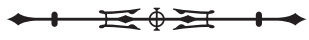


JURMAIN, Robert. Stress and the etiology of osteoarthritis. American Journal of Physical Anthropology, v. 46, n. 2, p. 353365, 1977.

KELLGREN, J. H.; LAWRENCE, J. S. Radiological assessment of osteo-arthrosis. Annals of the Rheumatic Diseases, v. 16, n. 4, p. 494-502, 1957.

KENNEDY, Kenneth. Skeletal markers of occupational stress. In: ISCAN, Mehmet; KENNETH, Kennedy (Eds.). Reconstruction of life from the skeleton. New York: Alan R. Liss, 1989. p. 129-160.

KLAUS, Haagen; SPENCER LARSEN, Clark; TAM, Manuel. Economic intensification and osteoarthritic joint disease: life and labor on the postcontact North Coast of Peru. American Journal of Physical Anthropology, v. 139, n. 2, p. 204-221, 2009.

KNEIP, Lina Maria. Cultura material e subsistência das populações pré-históricas de Saquarema, RJ. Rio de Janeiro: Museu Nacional/ UFRJ, 1994.

KNEIP, Lina Maria; MACHADO, Lilian Cheuiche. Os ritos funerários das populações pré-históricas de Saquarema, RJ: sambaquis da Beirada, Moa e Pontinha. Rio de Janeiro: Museu Nacional/UFRJ, 1993

KNÜSEL, Christopher. Bone adaptation and its relationship to physical activity in the past. In: COX, Margareth; MAYS, Simon (Eds.). Human osteology in archaeology and forensic science. Cambridge: Cambridge University Press, 2000. p. 381-401.

LEQUESNE, M.; DANG, N.; LANE, N. Sport practice and osteoarthritis of the limbs. Osteoarthritis and Cartilage, v. 5, n. 2. p. $75-86,1997$

LESSA, Andrea. Daily risks: a biocultural approach to acute trauma in pre-colonial coastal populations from Brazil. International Journal of Osteoarchaeology, v. 21, n. 2, p. 159-172, 2011.

LESSA, Andrea. Reflexões preliminares sobre paleoepidemiologia da violência em grupos ceramistas litorâneos: (I) Sítio Praia da Tapera - SC. Revista do Museu de Arqueologia e Etnologia, v. 15, p. 199-207, 2005

LESSA, Andrea; GASPAR, Maria Dulce. Estratégias de subsistência, complexidade social e violência entre grupos sambaquieiros do litoral brasileiro. In: MAZZ, José Lopes; BERÓN, Monica (Eds.). Indicadores arqueológicos de violência, guerra e conflito. Montevideo: Editorial Universitária. No prelo.

LESSA, Andrea; COELHO, Izaura. Lesões vertebrais e estilos de vida diferenciados em dois grupos sambaquieiros do litoral fluminense. Revista do Museu de Arqueologia e Etnologia, v. 20, p. 77-89, 2010.

LESSA, Andrea; SCHERER, Luciane Zanenga. O outro lado do paraíso: novos dados e reflexões sobre violência entre pescadorescoletores pré-coloniais. Revista do Museu de Arqueologia e Etnologia, v. 18, p. 89-100, 2008.
LIEVERSE, Angela; WEBER, Andrzej; BAZALIISKIY, Vladimir Ivanovick; GORIUNOVA, Olga Ivanova; SAVEL'EV, Nikolai Aleksandrovich. Osteoarthritis in Siberia's Cis-Baikal: skeletal indicators of hunter-gatherer adaptation and cultural change. American Journal of Physical Anthropology, v. 132, n. 1, p. 1-16, 2007.

LIEVENSE, A.; BIERNA-ZEINSTRA, S.; VERHAGEN, A.; VERHAAR, J.; KOES, B. Influence of work on the development of osteoarthritis of the hip: a systematic review. The Journal of Rheumatology, v. 28, n. 11, p. 2520-2528, 2001.

LIMA, Tania Andrade. Em busca dos frutos do mar: os pescadorescoletores do litoral centro-sul do Brasil. Revista da Universidade de São Paulo, v. 44, p. 270-327, 1999-2000

MACHADO, Lilia Cheuiche. Análise dos remanescentes ósseos humanos do sítio arqueológico Corondó, RJ: aspectos biológicos e culturais. Rio de Janeiro: Instituto de Arqueologia Brasileira, 1984

MERBS, Charles. Patterns of activity-induced pathology in a Canadian Inuit population. Ottawa: Archaeological Survey of Canada/National Museum of Man, 1983.

MOLNAR, Petra; TORBJORN, Ahlstrom; LEDEN, Ido. Osteoarthritis and activity - an analysis of the relationship between eburnation, Musculoskeletal Stress Markers (MSM) and age in two Neolithic hunter-gatherer populations from Gotland, Sweden. International Journal of Osteoarchaeology, v. 21, n. 3, p. 283-291, 2011.

NEVES, Walter. Incidência e distribuição de osteoartrites em grupos coletores do litoral do Paraná: uma abordagem osteobiográfica. Clio (Série Arqueológica), v. 6, p. 47-62, 1984.

OKUMURA, Maria Mercedes; EGGERS, Sabine. The people of Jabuticabeira II: reconstruction of the way of life in a Brazilian shellmound. HOMO - Journal of Comparative Human Biology, v. 55, n. 3, p. 263-281, 2005 .

ORTNER, Donald. Description and classification of degenerative bone changes in the distal joint surfaces of the humerus. American Journal of Physical Anthropology, v. 28, n. 2, p. 139-155, 1968.

PETRONILHO, Cecília Carlucci. Comprometimento articular como um marcador de atividades em um grande sambaquicemitério. 2005. Dissertação (Mestrado em Ciências Biológicas) - Universidade de São Paulo, São Paulo, 2005.

PONCE, Paola Vanessa. A comparative study of activity-related skeletal changes in 3rd-2nd millennium BC coastal fishers and 1 st millennium AD inland agriculturists in Chile, South America. 2010. Tese (Doutorado em Arqueologia) - Durham University, Durham, 2010.

PROUS, Andre. Arqueologia brasileira. Brasília: Universidade de Brasília, 1992.

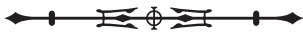


RESNICK, Donald; NIWAYAMA, Gen. Diagnosis of bone and joint disorders. Philadelphia: WB Saunders Company, 1988.

ROBIN, Joshua. A paleopathological assessment of osteoarthritis in the lower appendicular joints of individuals from the Kellis 2 cemetery in the Dakhleh Oasis, Egypt. 2008. Dissertação (Mestrado em Antropologia) - University of Central Florida, Orlando, 2008.

RODRIGUES-CARVALHO, Claudia. Marcadores de estresse ocupacional em populações sambaquieiras do litoral fluminense. 2004. Tese (Doutorado em Saúde Pública) - Escola Nacional de Saúde Pública/Fundação Oswaldo Cruz, Rio de Janeiro, 2004.

ROGERS, Julliet; DIEPPE, Paul. Is tibiofemoral osteoarthritis in the knee joint a new disease? Annals of the Rheumatic Diseases, $v$. 53, n. 9, p. 612-613, 1994.

ROHR, João Alfredo. Pesquisas arqueológicas em Santa Catarina: I - Exploração sistemática do sítio da Praia da Tapera. Pesquisas, Série Antropologia, v. 15, p. 3-20, 1966.

ROHR, João Alfredo. Pesquisas paleo-etnográficas na Ilha de Santa Catarina. Pesquisas, Série Antropologia, v. 3, p. 119-266, 1959.

SALTER, Donald. Degenerative joint disease. Current Diagnostic Pathology, v. 8, n. 1, p. 11-18, 2002.

SOSLOWSKY, L. J.; FLATOW, E. L.; BIGLIANI, L. U.; PAWLUK, R. J.; ATESHIAN, G. A.; MOW, V. C. Quantitation of in situ contact areas at the glenohumeral joint: a biomechanical study. Journal of Orthopaedic Research, v. 10, n. 4, p. 524-534, 1992.
SOUZA, Sheila Mendonça de. Estresse, doença e adaptabilidade: estudo comparativo de dois grupos pré-históricos em perspectiva biocultural. 1995. Tese (Doutorado em Saúde Pública) - Escola Nacional de Saúde Pública/Fundação Oswaldo Cruz, Rio de Janeiro, 1995.

WALDRON, Tony. Association between osteoarthritis of the hand and knee in a population of skeletons from London. Annals of the Rheumatic Diseases, v. 56, n. 2, p. 116-118, 1997.

WALDRON, Tony. Osteoarthritis in a Black Death cemetery in London. International Journal of Osteoarchaeology, v. 2, n. 3, p. 235-240, 1992.

WALDRON, Tony. Prevalence and distribution of osteoarthritis in a population from Georgian and early Victorian London. Annals of the Rheumatic Diseases, v. 50, n. 5, p. 301-307, 1991.

WEISS, Elizabeth; JURMAIN, Robert. Osteoarthritis revised: a contemporary review of aetiology. International Journal of Osteoarchaeology, v. 17, n. 5, p. 437-450, 2007.

WESOLOWSKI, Veronica. A prática da horticultura entre os construtores de sambaquis e acampamentos litorâneos da região da Baía de São Francisco, Santa Catarina: uma abordagem bioantropológica. 2000. Dissertação (Mestrado em Antropologia) Universidade de São Paulo, São Paulo, 2000.

WOO, Eun Jin; SCIULLI, Paul. Degenerative joint disease and social status in the Terminal Late Archaic Period (1000-500 B.C.) of Ohio. International Journal of Osteoarchaeology, v. 23, p. 529-544, 2013. 
